\title{
Respuesta de la vegetación al manejo por corte en fajas en un arbustal de Mulguraea tridens en Patagonia sur
}

\author{
Sabrina L. Billoni ${ }^{1 / 凶}$; Pablo L. Peri ${ }^{1,2,3} \&$ Héctor A. Bahamonde ${ }^{1,2}$ \\ ${ }^{1}$ Universidad Nacional de la Patagonia Austral. Unidad Académica Río Gallegos (UNPA-UARG). ${ }^{2}$ Instituto Nacional de \\ Tecnología Agropecuaria (INTA). ${ }^{3}$ Consejo Nacional de Investigaciones Científicas y Técnicas (CONICET).
}

\begin{abstract}
Resumen. En Santa Cruz, aproximadamente 2.83 millones de hectáreas conforman el matorral de mata negra, una estepa arbustiva dominada por el arbusto Mulguraea tridens, donde la ganadería ovina extensiva es la principal actividad productiva. En este trabajo se estudió el efecto de la aplicación de técnicas de manejo de corte en fajas removiendo la parte aérea de todos los arbustos. Los tratamientos evaluados fueron un arbustal sin intervenir (testigo) y fajas de corte de 4 y $8 \mathrm{~m}$ de ancho. Por un término de 3 años se midieron variables meteorológicas (humedad, precipitaciones, temperatura y viento), el efecto de los tratamientos sobre variables del suelo, capacidad de rebrote de los arbustos de $M$. tridens y aspectos relacionados al banco de semillas del suelo (BSS) como la germinación potencial y el reclutamiento de plántulas en el campo. La cobertura vegetal y productividad del pastizal fue evaluada en los años 1, 3 y 5 luego de aplicados los tratamientos. Los cortes en fajas aportaron entre 5700 y $8000 \mathrm{~kg} /$ ha de materia seca de broza a la superficie del suelo, lo que disminuyó su amplitud térmica entre 2 y $5{ }^{\circ} \mathrm{C}$ y aumentó su contenido de humedad entre 2.5 y $7.5 \%$ en los meses de primavera-verano. La productividad promedio del inter-coironal en el testigo fue $11.6 \mathrm{~kg} / \mathrm{ha}$ de materia seca, mientras que en las fajas de corte se obtuvieron hasta $88.2 \mathrm{~kg} / \mathrm{ha}$ de materia seca al quinto año de medición, posiblemente por un mejor aprovechamiento del agua del suelo ya que no se detectaron diferencias en el reclutamiento de las plántulas. No se observó un efecto significativo de los tratamientos sobre el BSS. Los arbustos mostraron capacidad de rebrotar desde las ramas, lo que brinda una mayor flexibilidad en el manejo a largo plazo y permite un esquema de rotación de los cortes en fajas.
\end{abstract}

[Palabras clave: suelo, vegetación, rebrote, arbusto, banco de semillas, germinación]

\begin{abstract}
AвsтRACт. Response of vegetation to management by cuttings along strips in a Mulguraea tridens shrubland in southern Patagonia. In Santa Cruz province, nearly 2.83 million hectares are occupied by a shrub steppe dominated by Mulguraea tridens (locally called matorral de mata negra), where the main activity is extensive sheep farming. This paper focuses on the effects of management practices such as removal in strips of the above ground shrub layer. The treatments were the control and the cutting of 4 and $8 \mathrm{~m}$ wide strips. Meteorological variables (humidity, rainfall, temperature and wind), the effect of treatments on soil variables, shrub regrowth capacity and aspects related to soil seed bank (SSB), potential germination and seedling recruitment in the field were monitored for a term of three years. The vegetation cover and grassland productivity was assessed after one, three and five years of treatments application. Shrub litter from cutting strip treatments varied between 5700 and $8000 \mathrm{~kg} / \mathrm{ha}$ of dry matter, resulting in a decrease in soil range temperature $\left(2-5^{\circ} \mathrm{C}\right)$ and an increase in soil moisture (2.5-7.5\%) during spring-summer months. Short grasses and herb productivity in the control was $11.6 \mathrm{~kg} / \mathrm{ha}$ of dry matter and under strips management was up to $88.2 \mathrm{~kg} / \mathrm{ha}$ of dry matter in the fifth year possibly due to better soil water use rather than seedling establishment. No significant effect of treatments on the SSB was observed. Shrubs showed ability to resprout, which provide flexibility in long term management allowing a rotational scheme of strips.
\end{abstract}

[Keywords: soil, vegetation, regrowth, shrub, seed bank, germination]

Editor asociado: Guillermo Martínez Pastur

$\triangle$ sbilloni230@yahoo.com.ar 


\section{INTRODUCCIÓN}

Las regiones áridas y semiáridas del mundo representan aproximadamente una tercera parte de la superficie continental (Whitford and Wade 2002), donde las gramíneas perennes y los arbustos son las principales formas de vida dominantes en tales ecosistemas (Bertiller et al. 2005). En la provincia de Santa Cruz, aproximadamente 2.83 millones de hectáreas conforman el área ecológica denominada matorral de mata negra, el principal ecosistema arbustivo de Patagonia sur. Su ubicación actual lo sitúa cerca de 130 establecimientos ganaderos. Este ecosistema está conformado por una estepa arbustiva de porte medio, de entre 40 y $70 \mathrm{~cm}$ de altura, dominada en un 60-70\% por Mulguraea tridens (Lag.) N. O'Leary and P. Peralta (mata negra), en algunos casos con distribución continua y en otros en forma de mosaicos con estepa graminosa de Pappostipa speciosa (Trin. and Rupr.) Romaschy P. chrysophylla(E. Desv.) Romasch. var. chrysophylla (coirones amargos) o Festuca pallescens (St. Yves) Parodi (coirón blanco). Ocupa mesetas y terrazas que llegan desde el nivel del mar hasta los $900 \mathrm{~m}$ s.n.m. al norte del río Santa Cruz, y entre éste y el río Coyle.

El valor máximo de cobertura de este arbusto es $\sim 70 \%$, y coincide con ambientes de menor disponibilidad de forraje para la cría de ovinos, en comparación con estepas graminosas. Si bien $M$. tridens es dominante, otrosarbustoscomoNardophyllumobtusifolium Hook. et Arn. y Berberis microphylla G. Forst. (calafate) pueden enriquecer este estrato (Oliva et al. 2001). En el inter-coironal, conformado por pastos cortos, dicotiledóneas herbáceas y ciperáceas preferidas por el ganado, se encuentran las especies Poa spiciformis(Steud.) Hauman and Parodi var. spiciformis, Bromus setifolius J. Presl var. setifolius, Carex argentina Barros, entre otros. Los campos de Patagonia sur en general fueron utilizados para la producción ganadera extensiva por más de 100 años.

Con el antecedente existente en la región de Magallanes de Chile (Covacevich and Doberti 1988), y aunque no se trata de las mismas especies, se considera que la práctica de remoción de la parte aérea de los arbustos podría ser una alternativa al manejo actual de los matorrales de $M$. tridens en Santa Cruz ya que aumentaría la disponibilidad forrajera y el manejo del rodeo de animales. Por otro lado, los arbustos desempeñan un rol fundamental en la dinámica de parches ya que actuarían como facilitadores en la instalación de nuevas plantas (Sala and Aguiar 1995). El banco de semillas del suelo (BSS) es un componente importante de la dinámica vegetal ya que el reclutamiento de nuevos individuos depende no sólo de condiciones favorables para la germinación y el establecimiento, sino también de la producción y la dispersión de semillas (Aguiar and Sala 1997). En ecosistemas semiáridos, el BSS presenta una alta heterogeneidad espacial a causa de los parches de la vegetación establecida (Aguiar and Sala 1999). La dinámica de las comunidades vegetales y sus respuestas frente a los disturbios (como la remoción de la biomasa aérea de arbustos) podrían ser mejor comprendidas teniendo en cuenta diferentes aspectos del BSS tales como su densidad y composición, su persistencia en el suelo y su similitud florística con la vegetación establecida (Márquez et al. 2002). El manejo de arbustales en Patagonia, a través de la remoción de su parte aérea distribuida espacialmente en fajas, podría afectar la dinámica del BSS. El objetivo de este trabajo fue estudiar la respuesta de la vegetación a la aplicación de cortes en fajas, analizando a los individuos de $M$. tridens y a la vegetación circundante, respecto de la germinación potencial del BSS y el reclutamiento de plántulas in situ, comparando con un arbustal sin intervención (testigo). Se plantearon las siguientes hipótesis de trabajo: i) los individuos de $M$. tridens rebrotan luego del corte de la parte aérea, lo que permitiría la recuperación del arbustal a lo largo del tiempo, y ii) los cortes en fajas de $M$. tridens incrementan el reclutamiento de plántulas y cobertura de especies forrajeras en el ecosistema arbustivo a partir de los cambios micro-ambientales a nivel del suelo (temperatura y humedad) en las áreas tratadas con respecto al testigo.

\section{Materiales y Métodos}

\section{Sitio de estudio}

El ensayo de cortes en fajas en el arbustal de $M$. tridens se realizó en la Ea. Chali Aike (5107'23.3" S - 7058'37.6" O), ubicada cerca de la zona del paraje La Esperanza, a la vera del río Coyle. El establecimiento cuenta con una superficie de 10000 ha, dominadas por estepa arbustiva. Está dividido en cuatro campos de 2500 ha cada uno, que son utilizados en época invernal por ganado ovino. El arbustal es denso y la altura de los arbustos predominantes es en promedio $0.8 \mathrm{~m}$. El clima es frío y árido, con temperaturas medias 
anuales entre 6.5 y $8.5^{\circ} \mathrm{C}$. Las lluvias anuales oscilan entre 150 y $200 \mathrm{~mm}$, y presentan un máximo invernal. Los vientos predominantes son del sector oeste-sudoeste. Los suelos son Aridisoles y Molisoles, profundos, de textura predominantemente arenosa y de buen drenaje, con gran presencia de canto rodado en el perfil (Godagnone y Salazar Lea Plaza 2004).

El área de estudio se sectorizó en tres subáreas, dentro de las cuales se distribuyeron al azar tres repeticiones de cada tratamiento (Figura 1). Los tratamientos considerados fueron: i) testigo: superficies ocupadas por $M$. tridens en estado natural (sin cortes), donde cada repetición abarcó $\sim 0.5$ ha, ii) faja de $8 \mathrm{~m}$ : donde se realizaron tres fajas de corte de $8 \mathrm{~m}$ de ancho x $200 \mathrm{~m}$ de largo, intercaladas con fajas de $4 \mathrm{~m}$ de ancho de arbustal sin intervenir (0.72 ha), lo que representa el 66\% de superficie total de arbustos removidos, y iii) faja de 4 $\mathrm{m}$ : donde se efectuaron tres fajas de corte de $\sim 4 \mathrm{~m}$ de ancho x $200 \mathrm{~m}$ de largo, intercaladas con fajas de $4 \mathrm{~m}$ de ancho de arbustal sin intervenir ( $0.48 \mathrm{ha})$, en donde la superficie total de arbustos removidos representó el 50\%. El corte de la parte aérea de los individuos de $M$. tridens se realizó a una altura de 5 a $8 \mathrm{~cm}$ del suelo en los tratamientos de corte.

Las fajas se orientaron en dirección nortesur, perpendicular a la dirección de los

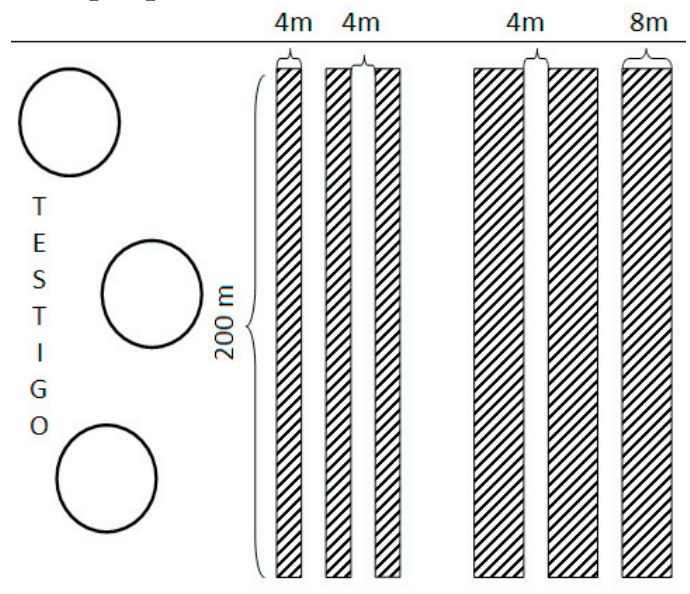

Figura 1. Esquema del diseño del ensayo de manejo de arbustales de Mulguraea tridens (Ea. Chali Aike) mediante técnicas de corte en fajas, ubicado en el área ecológica Matorral de Mata Negra, Santa Cruz. Los círculos indican las repeticiones del testigo, mientras que las zonas con rayas corresponden a las repeticiones de las fajas de corte de 4 y $8 \mathrm{~m}$.

Figure 1. Experimental design scheme of shrubland management study of Mulguraea tridens (Ea. Chali Aike) by cutting techniques in strips, located in the green area shrubland Mata Negra, Santa Cruz. The circles indicate the repetitions of the control, while areas with stripes correspond to repetitions of cutting strips of 4 and $8 \mathrm{~m}$. vientos predominantes. El trabajo de corte se realizó en el mes de agosto de 2009, cuando el suelo se encontraba congelado. Realizar este tipo de trabajos en épocas invernales facilitó el paso de la maquinaria y minimiza el impacto en el suelo. Para la aplicación de los tratamientos de corte se utilizó una picadora a martillos marca FALC Modelo CONDOR (Faenza, Italia) de un ancho de labor de $1.80 \mathrm{~m}$. Este tipo de maquinaria no produce remoción del suelo durante el trabajo de corte. Para permitir que las plantas nuevas se establezcan correctamente y poder evaluar el efecto de los tratamientos de corte respecto a las condiciones ambientales, se evitó el ingreso de hacienda desde el año 2009, mediante un alambrado perimetral (4 ha).

\section{Clima y suelos}

Las mediciones se realizaron desde septiembre de 2009 a mayo de 2012. La humedad relativa y la temperatura del aire (1.5 m de altura) y suelo ( $5 \mathrm{~cm}$ de profundidad) se registraron con sensores marca Hobo (USA). La humedad del suelo se midió con sensores marca Cavadevices (Argentina) ubicados a $5.0 \mathrm{~cm}$ de profundidad. Se definió esta profundidad para evaluar posibles efectos sobre la germinación de semillas y reclutamiento de plántulas. Los sensores se instalaron en los sectores medios de las fajas de corte de cada tratamiento y al azar en el testigo (1 sensor por tratamiento). La frecuencia de toma de datos fue cada $1 \mathrm{~h}$. Las precipitaciones se estimaron con un pluviómetro marca Cavadevices (Argentina), ubicado en el testigo a $1.5 \mathrm{~m}$ de altura.

La velocidad del viento se midió con un anemómetro manual (SIMS R-77C, USA) a $1.50 \mathrm{~m}$ de altura (velocidad del viento de referencia) y a $0.15 \mathrm{~m}$, en el sector medio de las fajas de corte en cada tratamiento y en claros en el testigo, a lo largo de las distintas estaciones del año. La reducción relativa (R1) de la velocidad del viento a $0.15 \mathrm{~m}$ (us) sobre el suelo en los tres tratamientos, se determinó en relación a la velocidad del viento medida por sobre el estrato arbustivo, a $1.5 \mathrm{~m}$ (uo) de altura (fuera de la influencia de los arbustos). La R1 se determinó de acuerdo a:

$\mathrm{R} 1=(\mathrm{uo}-\mathrm{us}) / \mathrm{uo}^{*} 100$

Los datos obtenidos se procesaron para calcular los valores medios de temperatura y humedad mínima, media y máxima diarias, y mensuales. Para caracterizar la textura y el 
contenido de nutrientes del suelo previo a la aplicación de los cortes se tomó una muestra compuesta por tres sub-muestras en zonas bajo arbustos y entre arbustos en los $15 \mathrm{~cm}$ superiores del suelo. A los 3 años, se tomó una muestra por tratamiento, para monitorear el nivel de nutrientes ( $\mathrm{N}$ y $\mathrm{P})$.

\section{Muestreos de los arbustos}

Previo a los tratamientos se realizó una descripción del estrato arbustivo tomando 10 arbustos de $M$. tridens al azar en cada repetición del testigo y tratamientos de corte, en los cuales se midió altura máxima, diámetro mayor, diámetro menor y diámetro basal del canopeo. Luego del paso de la máquina se midió en las fajas de corte el material vegetal incorporado como broza (principalmente residuos de ramas trituradas de $M$. tridens) mediante 6 parcelas circulares de $0.5 \mathrm{~m}^{2}$ establecidas al azar en cada repetición. El material recolectado se secó en estufa a 60 ${ }^{\circ} \mathrm{C}$ por $72 \mathrm{~h}$ hasta peso constante y luego se pesó con balanza de precisión $( \pm 0.001 \mathrm{~g})$ para estimar la incorporación de materia seca (MS) por unidad de superficie.

Luego del corte, en cada repetición de los cortes en faja se instalaron 2 clausuras $\left(2.2 \mathrm{~m}^{2}\right)$ en arbustos de $M$. tridens, separadas entre sí por $60 \pm 30$ m. Se midió, por única vez, el número y diámetro de las ramas remanentes con rebrote por arbusto. Para cuantificar el crecimiento anual del rebrote de los individuos de $M$. tridens, a fines de la estación de crecimiento (abril), durante 3 años (2010-2012) se midió en cada individuo de las clausuras la longitud de 3 rebrotes por cuadrante (NE, NO, SE, SO) para detectar si existe un efecto del viento en el crecimiento. También se determinó si los rebrotes provenían de las ramas remanentes o de la raíz. Para estimar el crecimiento anual de $M$. tridens en el testigo, se marcaron con una cinta de plástico 6 individuos (2 individuos por cada repetición del testigo) en los cuales se midió a fínes de la estación de crecimiento (abril) durante 3 años (2010-2012) el crecimiento de 3 brotes en cada cuadrante.

\section{Cobertura vegetal}

Para determinar los potenciales cambios en la vegetación, en cada repetición de los tratamientos se midió la cobertura inicial, previa a la aplicación de los tratamientos y su evolución temporal discriminando por estratos (arbustos, inter-coironal, subarbustos y coirones). El estrato inter-coironal comprendió las gramíneas bajas, graminoides y hierbas, mientras que el estrato coironal comprendió las gramíneas altas. El monitoreo de la composición botánica y la cobertura se realizó en el mes de diciembre (2010, 2012 y 2014) a través del método de puntos (Levy and Madden 1933) en transectas de $50 \mathrm{~m}$ de largo y con estimaciones cada $10 \mathrm{~cm}$ (500 puntos por transecta). Además, se observaron los cambios en la cobertura de criptógamas, suelo desnudo, mantillo, material muerto en pie y broza de $M$. tridens producido en los cortes. En el caso de los tratamientos de corte en fajas de 4 y $8 \mathrm{~m}$, las transectas se ubicaron en 3 posiciones: i) al oeste o sotavento de la faja con $M$. tridens, ii) en el centro, y iii) al este o barlovento de la faja cortada, para captar la variación espacial de cambios en la vegetación. En el testigo, las 3 transectas fueron distribuidas al azar. La productividad primaria neta aérea del inter-coironal se midió durante el máximo de producción de biomasa (diciembre 2010, 2012 y 2014) utilizando un marco de $0.2 \mathrm{~m}^{2}$. Se realizaron 3 cortes en cada repetición de cada tratamiento. Se secaron las muestras en horno a $60{ }^{\circ} \mathrm{C}$ por $72 \mathrm{~h}$ hasta peso constante y se calculó la productividad de materia seca por hectárea.

\section{Banco de semillas del suelo}

Para conocer la emergencia potencial del BSS del ensayo se utilizó un método indirecto de germinación o incubación (Simpson et al. 1989). Para esto se realizaron dos tomas de muestras (durante 3 años luego de aplicar los cortes en fajas) en diciembre y en marzo, para captar las semillas de especies con diferentes tiempos de producción y dispersión de semillas. Cada muestra consistió en 2 submuestras de suelo por repetición en cada tratamiento, tomadas con un marco de 20x15 $\mathrm{cm}$ y a $3 \mathrm{~cm}$ de profundidad. En el caso del tratamiento testigo, a partir del segundo año se incorporó un muestreo entre los arbustos y bajo los mismos, evitando zonas de excesiva acumulación y erosión. Estas sub-muestras tuvieron el objetivo de minimizar los errores relacionados con la gran heterogeneidad espacial que presentan los bancos de semillas (Thompson 1986). Tomar muestras de menor tamaño resultaría dificultoso debido a la pedregosidad presente en el área de estudio. Las muestras, sin disturbar, se colocaron el mismo día del muestreo, en cámara de germinación con un régimen de $12 \mathrm{~h}$ de luz y $12 \mathrm{~h}$ de oscuridad, a una temperatura de $23^{\circ} \mathrm{C}$ como condiciones de germinación (ISTA 2009) durante cuarenta y cinco días. Las mismas se regaron diariamente para mantenerlas a capacidad de campo y se determinó el número 
de plántulas. Las plántulas fueron clasificadas en monocotiledóneas y dicotiledóneas. La emergencia de los cotiledones fue el criterio utilizado para evaluar el éxito de la germinación (Gross 1990).

Para evaluar el reclutamiento de plántulas in situ y compararlo con la emergencia potencial se instalaron dos clausuras de $1.1 \times 0.2 \mathrm{~m}$ por repetición en cada tratamiento. A partir del segundo año de estudio, en el tratamiento testigo se contempló la variabilidad espacial incorporando clausuras (2 por sub-repetición) en los sectores abiertos del arbustal (entre arbustos) y en los sectores cerrados (bajo arbustos). Al finalizar la estación de crecimiento (abril de 2010 a 2012) se realizó el conteo de las nuevas plantas reclutadas, las que fueron clasificadas en monocotiledóneas y dicotiledóneas y luego removidas.

\section{Análisis estadístico}

Para comparar el crecimiento de los rebrotes de los arbustos cortados con el crecimiento del testigo se realizó un ANOVA con medidas repetidas en el tiempo con los valores obtenidos de los brotes y rebrotes de acuerdo a los cuadrantes (NO, SO, NE, SE), siendo las fechas de muestreo el factor intra-sujetos y cada tratamiento el factor inter-sujetos. Para comparar las medias se utilizó la prueba de Tukey $(P<0.05)$. De la misma manera se evaluó la cobertura absoluta del suelo. Para evaluar el efecto de los tratamientos sobre la productividad del inter-coironal se realizó un ANOVA, utilizando la prueba de Tukey para separar medias $(P<0.05)$. Para determinar el efecto de los tratamientos en la emergencia potencial del BSS se realizó una prueba de la mediana $(P<0.05)$, en la que la variable de respuesta fue la densidad de plántulas de cada tratamiento. Para detectar diferencias en el BSS de acuerdo a la estación primaveraverano se realizó un ANOVA considerando como factores la estación de crecimiento y el tratamiento empleando la prueba de Tukey $(P<0.05)$ para separar las medias. De la misma manera se evaluó la distribución espacial en el testigo. Para evaluar el efecto de los tratamientos en el reclutamiento de plántulas in situ se realizó un ANOVA con medidas repetidas en el tiempo, siendo las fechas de muestreo el factor intra-sujetos y cada tratamiento el factor inter-sujetos. Para la separación de medias, por no ser las varianzas homogéneas, se utilizó la prueba de Games Howell $(P<0.05)$ al detectar diferencias significativas entre tratamientos y al evaluar la distribución espacial del testigo. Para los análisis se utilizó el software estadístico IBM SPSS Statistics 20.

\section{Resultados}

\section{Clima y suelos}

La humedad relativa promedio del aire fue similar en los tres tratamientos, con valores extremos de $44 \%$ en diciembre y $71 \%$ en mayo. Los valores medios y mínimos de temperatura del aire fueron similares en los tratamientos. Mientras que la temperatura media fluctuó entre $-2.1{ }^{\circ} \mathrm{C}$ (junio) y $13.4^{\circ} \mathrm{C}$ (diciembre), la mínima tuvo un rango de $-8.5^{\circ} \mathrm{C}$ a 4.1 ${ }^{\circ} \mathrm{C}$. Respecto a la temperatura máxima del aire, durante los meses de otoño e invierno se observaron valores mayores en la faja de $4 \mathrm{~m}$ y menores en el testigo. La precipitación acumulada anual fluctuó desde $77.5 \mathrm{~mm}$ (año 2010) a 136.4 mm (año 2011), siendola media del período $107 \mathrm{~mm}$. Las mayores precipitaciones ocurrieron en primavera-verano. El rango de velocidad de viento fuera de la influencia de los arbustos fue de 7 a $30 \mathrm{~km} / \mathrm{h}$. La reducción relativa de la velocidad del viento fue mayor $(P<0.05)$ en el tratamiento testigo $(72 \pm 6 \%)$, con respecto a los tratamientos de corte en fajas de $4 \mathrm{~m}(64 \pm 8 \%)$ y $8 \mathrm{~m}(59 \pm 7 \%)$. Los valores medios mensuales de temperatura del suelo fueron similares para los tres tratamientos. En relación a los máximos, se observó que en enero y diciembre fueron más elevados en el testigo. En contraste, los valores mínimos fueron más elevados en la faja de $8 \mathrm{~m}$ en enero en relación a los otros dos tratamientos. En el testigo se detectó una tendencia de mayor amplitud térmica de las medias entre octubre y marzo, siendo la diferencia máxima $4.9^{\circ} \mathrm{C}$ en el mes de octubre $(P<0.05)$. La humedad media del suelo fluctuó entre 10.8 y $27.8 \%$ dependiendo del tratamiento y de la época del año. La faja de $8 \mathrm{~m}$ mostró los valores más altos desde enero hasta junio, mientras que las fajas de $4 \mathrm{~m}$ y $8 \mathrm{~m}$ mostraron valores por encima del testigo desde septiembre hasta diciembre.

Antes de la aplicación de los tratamientos, el suelo presentó una textura franco a francoarenosa de reacción ligeramente ácida bajo arbustos y entre arbustos. El valor promedio de carbono orgánico fue $2.5 \%$ y el de $\mathrm{N}$ total fue $0.19 \%$, con una relación C/N de 12.7. Luego de tres años de efectuados los tratamientos de corte en fajas, la relación $\mathrm{C} / \mathrm{N}$ promedio del testigo fue 13, y 9 en los tratamientos de corte en fajas. El contenido de P varió entre 22.8 y $27.4 \mathrm{mg} / \mathrm{kg}$, y no se observaron problemas de salinidad (>1000 ohm/cm). 
Tabla 1. Cobertura absoluta del suelo $(\% \pm \mathrm{DE})$ en el ecosistema de arbustal de $M$. tridens (testigo) y bajo manejo con dos anchos de corte en fajas, a uno, tres y cinco años de aplicado el tratamiento. Letras minúsculas diferentes indican diferencias significativas entre tratamientos dentro de un mismo año y letras mayúsculas indican diferencias significativas entre años dentro de un mismo tratamiento $(P<0.05)$. CVT: cobertura vegetal total.

Table 1. Absolute ground cover $(\% \pm \mathrm{SD})$ in the $M$. tridens shrubland ecosystem (control) and under management with two intensities cut in strips after one, three and five years of treatment had been applied. Different lower case letters indicate significant differences between treatments in the same year and capital letters indicate significant differences between years in the same treatment $(P<0.05)$. CVT: total vegetation cover.

\begin{tabular}{|c|c|c|c|c|c|c|c|c|c|}
\hline \multicolumn{10}{|c|}{ Años transcurridos desde la aplicación del tratamiento } \\
\hline & \multicolumn{3}{|c|}{1 año } & \multicolumn{3}{|c|}{3 años } & \multicolumn{3}{|c|}{5 años } \\
\hline & Testigo & Faja de $4 \mathrm{~m}$ & Faja de $8 \mathrm{~m}$ & Testigo & Faja de $4 \mathrm{~m}$ & Faja de $8 \mathrm{~m}$ & Testigo & Faja de $4 \mathrm{~m}$ & Faja \\
\hline $\begin{array}{l}\text { u e } 1 \text { o } \\
\text { esnudo }\end{array}$ & $28.5 \pm 5.0 \mathrm{aA}$ & & & & & & & & \\
\hline Manti & $4.4 \pm 2.8 \mathrm{aA}$ & $3.2 \pm 2.4 \mathrm{aA}$ & $4.2 \pm 0.2 \mathrm{aA}$ & $3.2 \pm 0.7 \mathrm{aA}$ & $5.6 \pm 1.6 \mathrm{aA}$ & $5.4 \pm 0.7 \mathrm{aA}$ & $3.8 \pm 0.6 \mathrm{aA}$ & $6.8 \pm 1.8 \mathrm{aA}$ & $5.9 \pm 0.8 \mathrm{aA}$ \\
\hline Broza & - & $59.1 \pm 8.6 \mathrm{aA}$ & $56.3 \pm 1.1 \mathrm{aA}$ & 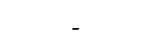 & $42.4 \pm 2.7 \mathrm{aB}$ & $45.4 \pm 3.8 \mathrm{aB}$ & - & $38.5 \pm 3.2 \mathrm{aB}$ & $=3.2 \mathrm{aB}$ \\
\hline $\begin{array}{l}\text { Muerto } \\
\text { en pie }\end{array}$ & $1.4 \pm 0.3 \mathrm{aA}$ & $1.0 \pm 0.1 \mathrm{aA}$ & $1.3 \pm 0.3 \mathrm{aA}$ & $0.9 \pm 0.3 \mathrm{aB}$ & $1.4 \pm 0.3 \mathrm{aB}$ & $2.3 \pm 0.2 \mathrm{bB}$ & $1.2 \pm 0.3 \mathrm{aB}$ & $1.6 \pm 0.4 \mathrm{aB}$ & $1.9 \pm 0.3 \mathrm{bB}$ \\
\hline $\begin{array}{l}\text { Criptó- } \\
\text { gamas }\end{array}$ & $0.5 \pm 0.4 \mathrm{aA}$ & $0.5 \pm 0.1 \mathrm{aA}$ & $0.5 \pm 0.4 \mathrm{aA}$ & $0.0 \pm 0.0 \mathrm{aA}$ & $0.2 \pm 0.2 \mathrm{aA}$ & $0.0 \pm 0.1 \mathrm{aA}$ & $0.2 \pm 0.1 \mathrm{aA}$ & $0.3 \pm 0.2 \mathrm{aA}$ & $0.1 \pm 0.1 \mathrm{aA}$ \\
\hline Arbusto & $36.2 \pm 4.4 \mathrm{aA}$ & $4.6 \pm 1.2 \mathrm{bA}$ & $5.2 \pm 2.5 \mathrm{bA}$ & $38.6 \pm 1.9 \mathrm{aA}$ & $7.2 \pm 0.7 \mathrm{bA}$ & $4.6 \pm 1.5 \mathrm{bA}$ & $39.2 \pm 1.9 \mathrm{aA}$ & $9.8 \pm 0.9 \mathrm{bA}$ & $5.8 \pm 1.8 \mathrm{bA}$ \\
\hline Coirón & $8.1 \pm 2.8 \mathrm{aA}$ & $4.3 \pm 2.1 \mathrm{aA}$ & $4.0 \pm 1.8 \mathrm{aA}$ & $7.0 \pm 1.0 \mathrm{aA}$ & $7.1 \pm 2.0 \mathrm{aA}$ & $6.4 \pm 3.0 \mathrm{aA}$ & $7.5 \pm 1 \mathrm{aA}$ & $2.5 \mathrm{aA}$ & $.5 \mathrm{aA}$ \\
\hline In t e r - & $8.2 \pm 0.6 \mathrm{aA}$ & $4.1 \pm 1.4 \mathrm{bA}$ & $4.5 \pm 0.8 \mathrm{bA}$ & $7.2 \pm 1.3 \mathrm{aA}$ & $7.4 \pm 0.8 \mathrm{aB}$ & $7.2 \pm 1.0 \mathrm{aA}$ & $8.5 \pm 1.3 \mathrm{aA}$ & $12.7 \pm 1.1 \mathrm{aB}$ & $9.7 \pm 1.4 \mathrm{aA}$ \\
\hline $\begin{array}{l}\mathrm{S} \text { u b } \\
\text { arbusto }\end{array}$ & $12.7 \pm 0.3 \mathrm{aA}$ & $7.3 \pm 1.5 \mathrm{bA}$ & $9.0 \pm 1.2 \mathrm{bA}$ & $8.9 \pm 0.9 \mathrm{aA}$ & $11.7 \pm 1.9 \mathrm{abB}$ & $13.7 \pm 1.0 \mathrm{bB}$ & $9.1 \pm 0.9 \mathrm{aA}$ & $8.9 \pm 1.3 \mathrm{abB}$ & $13.1 \pm 1.0 \mathrm{bB}$ \\
\hline CVT & $65.2 \pm 2.5 \mathrm{aA}$ & $20.3 \pm 5.9 \mathrm{bA}$ & $22.5 \pm 1.9 \mathrm{bA}$ & $61.8 \pm 1.2 \mathrm{aA}$ & $33.4 \pm 2.1 \mathrm{bB}$ & $31.8 \pm 5.0 \mathrm{bB}$ & $64.3 \pm 7.3 \mathrm{aA}$ & $40.0 \pm 5.1 \mathrm{bB}$ & $35.9 \pm 4.9 \mathrm{bB}$ \\
\hline
\end{tabular}

Muestreos de los arbustos

La altura máxima promedio de los individuos de mata negra antes de la intervención fue $0.81 \pm 0.11 \mathrm{~m}$, y los diámetros mayor y menor del canopeo fueron $1.7 \pm 0.2 \mathrm{~m}$ y $1.5 \pm 0.2$ $\mathrm{m}$, respectivamente. El diámetro basal de los arbustos fue $0.3 \pm 0.1 \mathrm{~m}$. Luego de la intervención se determinó que los rebrotes no provinieron de la base del arbusto sino de las ramas remanentes, cuyo diámetro medio fue $1.7 \pm 0.4 \mathrm{~cm}$. La cantidad de ramas por arbusto que presentaron rebrote fue $22 \pm 4.8$, con una densidad de 50 rebrotes $/ \mathrm{m}^{2}$. El número de rebrotes y el crecimiento de los mismos fue similar en los cuadrantes (ANOVA, $\mathrm{gl}=2, F=0.538, P>0.05)$.El crecimiento anual promedio de los brotes de las matas testigo (años 2010 a 2012) fue de $1.8 \mathrm{~cm}$, mientras que en los tratamientos de corte en faja, el crecimiento promedio de los rebrotes para el mismo período fue mayor (ANOVA, $\mathrm{gl}=2$, $F=87.8, P<0.001)$, con un valor de $4.9 \mathrm{~cm}$. Además, el crecimiento promedio del último año en los tratamientos de corte en faja fue de $7.1 \mathrm{~cm}$, siendo mayor a los dos primeros años (ANOVA, $\mathrm{gl}=1, F=37.9, P<0.001$ ). Finalmente, el material incorporado al suelo como broza fue $5747 \pm 3892 \mathrm{~kg} /$ ha de MS para la faja de 4 $\mathrm{m}$, y de $7995 \pm 4990 \mathrm{~kg} / \mathrm{ha}$ de MS para la faja de $8 \mathrm{~m}$.

\section{Cobertura vegetal}

Previo a los cortes, la cobertura vegetal absoluta fue de $65.2 \%$. Luego de la aplicación de los tratamientos, la cobertura disminuyó a $20.3 \%$ y $22.5 \%$ en los tratamientos de corte de 4 y $8 \mathrm{~m}$, respectivamente (ANOVA, $\mathrm{gl}=2$, $F=232.7, P<0.001)$. A los 3 y 5 años de aplicados los tratamientos se mantuvo la diferencia en cobertura vegetal absoluta $(P<0.001)$ (Tabla 1). Por otra parte, desde el primer año, se observó una disminución del porcentaje de suelo desnudo en las fajas en relación al testigo (Tabla 1) como consecuencia del aumento del residuo de $M$. tridens (mayor al $50 \%$ en el primer año). Al comienzo del ensayo, previo a la aplicación de los tratamientos, la riqueza específica fue de 20 especies vegetales vasculares (Tabla 2). Las especies $M$. tridens, P. chrysophylla y el sub-arbusto Clinopodium darwinii (Benth.) Kuntze contribuyeron al 80\% de la CR de la vegetación.

La producción de biomasa aérea del intercoironal fue mayor $(P<0.05)$ en las fajas respecto al testigo luego de 1, 3 y 5 años de aplicados los tratamientos. La productividad media del testigo fue $11.4 \mathrm{~kg} / \mathrm{ha}$ de MS, mientras que el promedio de la productividad en los tratamientos de corte en faja fue $25 \mathrm{~kg} /$ ha de MS (año 2010), $43 \mathrm{~kg} / \mathrm{ha}$ de MS (año 2012) y $72 \mathrm{~kg} / \mathrm{ha}$ de MS (año 2014).

\section{Banco de semillas del suelo}

En la Tabla 3 se presentan los valores (plántulas $/ \mathrm{m}^{2}$ ) de emergencia potencial del banco de semillas del suelo. La cantidad de plántulas emergidas bajo condiciones óptimas de temperatura y humedad del BSS estuvo 


\begin{tabular}{|c|c|c|c|c|c|c|}
\hline \multicolumn{4}{|l|}{ Especie } & Estrato & $\begin{array}{l}\text { Cobertura } \\
\text { relativa }\end{array}$ & \\
\hline \multicolumn{4}{|c|}{ Acaena poeppigiana Gay } & IC & $0.4 \pm 0.5$ & \\
\hline \multicolumn{4}{|c|}{ Azorella sp. } & SA & $0.1 \pm 0.1$ & \\
\hline \multicolumn{4}{|c|}{ Berberis microphylla G. Forst } & A & $1.2 \pm 2.2$ & \\
\hline \multicolumn{4}{|c|}{ Bromus setifolius J. Presl var. setifolius } & IC & $1.3 \pm 1.1$ & \\
\hline \multicolumn{4}{|c|}{ Calandrinia sp. } & IC & $0.1 \pm 0.1$ & \\
\hline \multicolumn{4}{|c|}{ Carex argentina Barros } & IC & $0.1 \pm 0.2$ & \\
\hline \multicolumn{4}{|c|}{ Cerastium arvense $\mathrm{L}$. } & IC & $0.3 \pm 0.2$ & \\
\hline \multicolumn{4}{|c|}{ Clinopodium darwinii (Benth.) Kuntze } & SA & $14.2 \pm 1.8$ & \\
\hline \multicolumn{4}{|c|}{ Colobanthus lycopodioides Griseb. } & SA & $0.3 \pm 0.2$ & \\
\hline \multicolumn{4}{|c|}{ Ephedra frustillata Miers } & SA & $0.5 \pm 0.4$ & \\
\hline \multicolumn{4}{|c|}{ Festuca pyrogea Speg. } & IC & $1.6 \pm 1.4$ & \\
\hline \multicolumn{4}{|c|}{ Mulguraea tridens (Lag.) N. O'Leary and P. Peralta } & A & $54.2 \pm 1.2$ & \\
\hline \multicolumn{4}{|c|}{ Nardophyllum brioides (Lam.) Cabrera } & SA & $0.6 \pm 0.3$ & \\
\hline \multicolumn{4}{|c|}{ Nassauvia aculeata (Less.) Poepp. and Endl. var. aculeata } & SA & $2.4 \pm 1.7$ & \\
\hline \multicolumn{4}{|c|}{ Nassauvia glomerulosa (Lag. ex Lindl.) D. Don } & SA & $1.1 \pm 1.8$ & Tabla 2. Cobertura relativa de \\
\hline \multicolumn{4}{|c|}{ Pappostipa chrysophylla (E. Desv.) Romasch. var. chrysophylla } & $\mathrm{C}$ & $12.3 \pm 3.1$ & especies $(\% \pm D S)$ en el ecosistema \\
\hline \multicolumn{4}{|c|}{ Pappostipa ibari (Phil.) Romasch. } & IC & $0.3 \pm 0.6$ & $\begin{array}{l}\text { de arbustal de } M \text {. tridens } \\
\text { (testigo), previo a la aplicación }\end{array}$ \\
\hline \multicolumn{4}{|c|}{ Perezia recurvata (Vahl) Less. ssp. recurvata } & SA & $0.4 \pm 0.4$ & de los tratamientos. \\
\hline \multirow{2}{*}{\multicolumn{4}{|c|}{$\begin{array}{l}\text { Poa spici formis (Steud.) Hauman and Parodi var. spiciformis } \\
\text { Trisetum cumingii (Steud.) Parodi ex Nicora }\end{array}$}} & IC & $8.5 \pm 2.2$ & Table 2. Relative species \\
\hline & & & & IC & $0.1 \pm 0.1$ & $\begin{array}{l}\text { cover }(\% \pm \mathrm{SD}) \text { in the } M \text {. tridens } \\
\text { shrubland ecosvstem before }\end{array}$ \\
\hline \multicolumn{6}{|c|}{$\mathrm{A}=$ arbusto; $\mathrm{C}=$ coirón; $\mathrm{IC}=$ inter-coironal; $\mathrm{SA}=$ subarbusto } & treatments application. \\
\hline \multirow{4}{*}{ Diciembre 2009} & Tratamiento & Monocotiledóneas & Dicotiled & óneas & Total & \\
\hline & Testigo & $111.1 \pm 14.7 \mathrm{a}$ & $78.8 \pm 24$ & $4.3 \mathrm{a}$ & $189.9 \pm 38.9 \mathrm{ab}$ & \\
\hline & Faja $4 \mathrm{~m}$ & $28.8 \pm 20.0 \mathrm{a}$ & $116.7 \pm 50$ & $0.9 \mathrm{ab}$ & $145.5 \pm 53.0 \mathrm{a}$ & \\
\hline & Faja $8 \mathrm{~m}$ & $133.3 \pm 67.4 \mathrm{a}$ & $294.4 \pm 7$ & $0.9 \mathrm{~b}$ & $427.7 \pm 65.5 b$ & de plántulas (plántulas $/ \mathrm{m}^{2} \pm E S$ ) \\
\hline \multirow[t]{3}{*}{ Marzo 2010} & Testigo & $105.6 \pm 40.1 \mathrm{a}$ & $89.9 \pm 38$ & $8.9 \mathrm{a}$ & $195.5 \pm 53.0 \mathrm{a}$ & del banco de semillas del suelo \\
\hline & Faja $4 \mathrm{~m}$ & $138.9 \pm 84.1 \mathrm{a}$ & $256.6 \pm 39$ & $94.4 \mathrm{a}$ & $395.5 \pm 194.7 \mathrm{a}$ & $\begin{array}{l}\text { del ecosistema de arbustal de } M \text {. } \\
\text { tridens (Testigo) y bajo maneio }\end{array}$ \\
\hline & Faja $8 \mathrm{~m}$ & $72.2 \pm 33.8 \mathrm{a}$ & $127.8 \pm 6$ & $2.6 \mathrm{a}$ & $200.0 \pm 83.9 \mathrm{a}$ & con dos anchos de corte en \\
\hline \multirow[t]{3}{*}{ Diciembre 2010} & Testigo & $41.7 \pm 12.7 \mathrm{a}$ & $38.9 \pm 10$ & $0.2 \mathrm{a}$ & $80.6 \pm 22.2 \mathrm{a}$ & fajas, durante tres años desde la \\
\hline & Faja $4 \mathrm{~m}$ & $38.9 \pm 20.0 \mathrm{a}$ & $255.6 \pm 11$ & $19.5 \mathrm{a}$ & $294.1 \pm 106.0 \mathrm{a}$ & cada fecha, letras distintas entre \\
\hline & Faja $8 \mathrm{~m}$ & $66.7 \pm 34.7 \mathrm{a}$ & $305.6 \pm 9$ & $0.9 \mathrm{a}$ & $372.3 \pm 121.8 \mathrm{a}$ & tratamientos indican diferencias \\
\hline \multirow[t]{3}{*}{ Marzo 2011} & Testigo & $13.9 \pm 2.8 \mathrm{a}$ & $100.0 \pm 5$ & $0.0 \mathrm{a}$ & $113.9 \pm 51.5 \mathrm{a}$ & \\
\hline & Faja $4 \mathrm{~m}$ & $16.7 \pm 9.6 \mathrm{a}$ & $94.4 \pm 78$ & $8.4 \mathrm{a}$ & $111.1 \pm 77.8 \mathrm{a}$ & Table 3. Potential seedlings \\
\hline & Faja $8 \mathrm{~m}$ & $22.2 \pm 11.1 \mathrm{a}$ & $105.6 \pm 4$ & $9.4 \mathrm{a}$ & $127.8 \pm 58.0 \mathrm{a}$ & of soil seed bank in the $M$. tridens \\
\hline Diciembre 2011 & Testigo & $61.1 \pm 42.0 \mathrm{a}$ & $144.4 \pm 6$ & $6.7 \mathrm{a}$ & $205.5 \pm 39.2 \mathrm{~b}$ & shrubland ecosystem (control) \\
\hline & Faja $4 \mathrm{~m}$ & $22.2 \pm 14.7 \mathrm{a}$ & $44.4 \pm 11$ & $1.1 \mathrm{a}$ & $66.6 \pm 9.6 \mathrm{a}$ & and under management with \\
\hline & Faja $8 \mathrm{~m}$ & $16.7 \pm 16.7 \mathrm{a}$ & $116.7 \pm 2$ & $5.5 \mathrm{a}$ & $133.3 \pm 34.7 \mathrm{ab}$ & $\begin{array}{l}\text { two intensities cut in strips } \\
\text { during three years from the }\end{array}$ \\
\hline Marzo 2012 & Testigo & $47.2 \pm 34.8 \mathrm{a}$ & $44.4 \pm 22$ & $2.7 \mathrm{a}$ & $91.6 \pm 45.9 \mathrm{a}$ & intervention (2010-2012). For \\
\hline & Faja $4 \mathrm{~m}$ & $133.3 \pm 69.6 \mathrm{a}$ & $62.5 \pm 28$ & $8.9 \mathrm{a}$ & $195.8 \pm 94.9 \mathrm{a}$ & $\begin{array}{l}\text { each date, aifferent letter } \\
\text { indicate significant difference }\end{array}$ \\
\hline & Faja $8 \mathrm{~m}$ & $83.3 \pm 61.1 \mathrm{a}$ & $50.0 \pm 34$ & $4.7 \mathrm{a}$ & $134.3 \pm 62.6 \mathrm{a}$ & between treatments $(P<0.05)$. \\
\hline
\end{tabular}

comprendida entre 66 y 427 plántulas $/ \mathrm{m}^{2}$. No se encontraron diferencias al evaluar la emergencia potencial a lo largo del tiempo para cada tratamiento $(P>0.05)$. Al agrupar y comparar los valores de emergencia potencial entre estaciones (primavera y verano) se encontró que la emergencia potencial de plántulas fue menor en verano (ANOVA, $\mathrm{gl}=1, F=4.1, P<0.05)$ en el tratamiento de corte en fajas de $8 \mathrm{~m}$. La distribución espacial de la emergencia potencial total entre los arbustos y bajo los mismos en el testigo no presentó diferencias significativas $(P>0.05)$. Tampoco se detectaron diferencias al discriminar por momento de producción de semillas (diciembre y marzo), o por monocotiledóneas y dicotiledóneas.

El reclutamiento de plántulas in situ fue similar entre tratamientos en cada año de medición (ANOVA, $\mathrm{gl}=2, F=1.2, P>0.05$ ) (Tabla 4). En el testigo y en la faja de $4 \mathrm{~m}$ hubo un aumento de la densidad total de plántulas establecidas en el tercer año de estudio 
Tabla 4. Reclutamiento de plántulas (plántulas $/ \mathrm{m}^{2} \pm$ ES) in situ (Ea. Chali Aike) del ecosistema de arbustal de mata negra (testigo) y bajo manejo con dos anchos de corte en fajas, medidos en el mes de abril a lo largo de tres temporadas luego de aplicados los tratamientos (20102012). Letras distintas indican diferencias significativas entre años para un mismo tratamiento $(P<0.05)$.

Table 4. Recruitment of seedlings (seedlings $/ \mathrm{m}^{2} \pm$ SE) in situ (Ea. Chali Aike) in the M. tridens shrubland ecosystem (control) and under management with two intensities cut in strips, measured in April over three seasons after applied treatments (2010-2012). Different letters indicate significant differences between years for the same treatment $(P<0.05)$.

\begin{tabular}{lllcc}
\hline Abril & Testigo & $1.5 \pm 1.5 \mathrm{~A}$ & $0.0 \pm 0.0 \mathrm{~A}$ & $1.5 \pm 1.5 \mathrm{~A}$ \\
2010 & Faja $4 \mathrm{~m}$ & $3.0 \pm 2.0 \mathrm{~A}$ & $2.3 \pm 2.3 \mathrm{~A}$ & $5.3 \pm 4.2 \mathrm{~A}$ \\
& Faja $8 \mathrm{~m}$ & $3.0 \pm 0.8 \mathrm{~A}$ & $0.0 \pm 0.0 \mathrm{~A}$ & $3.0 \pm 0.8 \mathrm{~A}$ \\
Abril & Testigo & $0.0 \pm 0.0 \mathrm{~A}$ & $3.8 \pm 3.2 \mathrm{~A}$ & $3.8 \pm 3.2 \mathrm{~A}$ \\
2011 & Faja $4 \mathrm{~m}$ & $6.1 \pm 0.8 \mathrm{~A}$ & $10.6 \pm 4.0 \mathrm{~B}$ & $16.7 \pm 4.6 \mathrm{~A}$ \\
& Faja $8 \mathrm{~m}$ & $9.8 \pm 4.6 \mathrm{~A}$ & $3.0 \pm 0.8 \mathrm{~A}$ & $12.8 \pm 4.0 \mathrm{~A}$ \\
Abril & Testigo & $11.0 \pm 3.1 \mathrm{~B}$ & $33.7 \pm 8.3 \mathrm{~B}$ & $44.7 \pm 7.2 \mathrm{~B}$ \\
2012 & Faja 4 m & $27.3 \pm 10.4 \mathrm{~B}$ & $28.0 \pm 8.7 \mathrm{C}$ & $55.3 \pm 19.0 \mathrm{~B}$ \\
& Faja $8 \mathrm{~m}$ & $14.4 \pm 4.6 \mathrm{~B}$ & $9.8 \pm 2.7 \mathrm{~A}$ & $24.2 \pm 3.0 \mathrm{~A}$
\end{tabular}

$(P<0.05)$, en relación a los dos primeros. En el reclutamiento de dicotiledóneas se observó un aumento en el testigo en el último año de medición, mientras que en la faja de $4 \mathrm{~m}$ el aumento se produjo en los dos últimos años de mediciones. La distribución espacial de la emergencia de plántulas in situ (total, monocotiledóneas y dicotiledóneas) no presentó diferencias significativas ( $P>0.05)$, considerando las situaciones entre arbustos y bajo arbustos en el testigo. Sin embargo, se observaron diferencias en el reclutamiento de plántulas entre años $(P<0.05)$ en ambas situaciones. En abril de 2011, la densidad de plántulas establecidas fue $0.8 \pm 0.8$ plántulas / $\mathrm{m}^{2}$ en la situación bajo arbustos y $6.8 \pm 5.7$ en la situación entre arbustos, mientras que en abril de 2012, las densidades fueron $56 \pm 8$ y $33.3 \pm 10.6$ plántulas $/ \mathrm{m}^{2}$, respectivamente.

\section{DISCUSIÓN}

\section{Clima y suelos}

Los valores de precipitación durante el período en el que se desarrolló el estudio del BSS estuvieron por debajo del valor promedio para la zona entre 2000 y 2010 (160 mm/año, datos de Ea. Don Ceferino), lo cual pudo tener influencia en la información generada, relacionada a los procesos de reclutamiento de la vegetación. En cuanto al efecto sobre la modificación de la velocidad del viento, la aplicación de los cortes en fajas produjo una disminución del efecto protector del arbustal contra el viento. Si bien no existen antecedentes bibliográficos de esta variable de estudio en este tipo de ambiente, en Patagonia sur ha sido extensamente estudiado el comportamiento del viento con respecto al efecto protector de las cortinas forestales (Peri, 2011) y los factores estructurales de la vegetación que determinan la reducción de la velocidad del viento. En el caso del arbustal natural, la estructura (densidad de arbustos, altura media y distancia entre individuos) determina una mayor reducción de la velocidad del viento. Sin embargo, es importante realizar estudios que determinen potenciales pérdidas de suelo en las fajas, respecto al matorral sin intervenir, debido al aumento de la velocidad del viento, el ingreso de animales y por precipitaciones torrenciales que ocurren cada 50-100 años.

En cuanto a la temperatura del suelo, la disminución de la amplitud térmica en las fajas podría explicarse, por un lado, como consecuencia de la protección de la broza hacia la incidencia directa de los rayos solares sobre el suelo, principalmente en los meses estivales, lo cual produce una disminución de la temperatura máxima. Por otra parte, en épocas invernales, la broza sobre la superficie del suelo actúa como trampa de la capa de aire aislando al suelo de las bajas temperaturas (Waring and Schlesinger 1985). El efecto de diversas coberturas vegetales sobre la amortiguación de la amplitud térmica fue documentado en bosques de Patagonia (Bahamonde et al. 2012), aunque no directamente relacionado a la cobertura por broza.

En relación a la humedad del suelo, la tendencia registrada de mayores valores en las fajas (especialmente la de $8 \mathrm{~m}$ ) respecto al testigo puede deberse a una reducción de la evaporación ya que la broza permite retener por un período mayor la humedad en el suelo. Efectos similares fueron reportados por Sharafatmandrad et al. (2010) en ambientes áridos de Irán. Sin embargo, en estos ambientes áridos de estepa la precipitación se caracteriza por una elevada variabilidad interanual. Es posible que la profundidad del suelo en la que se realizaron las mediciones $(5 \mathrm{~cm})$ de humedad del suelo no haya sido sensible a las respuestas de las plantas (especialmente el estrato inter-coironal) respecto del régimen de precipitaciones, que se caracteriza por el predominio de eventos discretos de poco volumen $(<10 \mathrm{~mm})$ (Sala and Lauenroth 1982; Golluscio et al. 1998). Prueba de esto surge del trabajo de Peri et al. (2011), quienes en el mismo ensayo, a partir de la medición de la conductividad estomática, detectaron que 
las plantas de $P$. spiciformis presentaron un mayor estatus hídrico en las fajas debido a la presencia de residuos de $M$. tridens.

\section{Muestreos de los arbustos}

La capacidad de rebrote de los arbustos determina la durabilidad en el tiempo de los cortes aplicados y de la capacidad de recuperación del arbustal. Dado que los individuos de $M$. tridens son capaces de rebrotar desde las ramas, se puede prever que la práctica de manejo de corte en fajas del arbustal es apropiada para asegurar su retorno a un estado similar al inicial. Además, esta capacidad de rebrote permite planificar un esquema de rotación de los cortes en fajas luego de la recuperación de las fajas cortadas inicialmente. Dado el valor promedio de crecimiento de los rebrotes $(4.9 \mathrm{~cm} / \mathrm{año})$, se esperaría que en el término de 16 años se recupere la altura media inicial del arbustal $(0.8 \mathrm{~m})$. El intervalo de tiempo de corte de las fajas dependerá de las necesidades de uso y de la altura óptima de los individuos de $M$. tridens para permitir un tránsito adecuado de los animales.

\section{Cobertura vegetal}

Un efecto importante de la aplicación de los tratamientos de corte en fajas fue la disminución del porcentaje de suelo desnudo en relación al testigo, ya que se redujo prácticamente a la mitad como consecuencia del aumento de broza de $M$. tridens resultante del corte. Por otra parte, Allegretti et al. (1997) informaron que en Nacuñán (Mendoza), bajo tratamiento mecánico de control de arbustos, hubo una reducción de la cobertura de arbustos por al menos tres años luego de la aplicación de los tratamientos. En general, el manejo de arbustales aumenta la cobertura y la producción de gramíneas. Por ejemplo, la práctica de rolado (técnica que consiste en pasar un cilindro metálico con cuchillas cortante, que a su paso aplasta y corta la vegetación existente y cuyas cuchillas pueden penetrar $15-25 \mathrm{~cm}$ en el suelo), redujo de manera efectiva la cobertura de las leñosas y aumentó significativamente la productividad del pastizal en La Pampa (Adema et al. 2003). La respuesta de los métodos de remoción de arbustos sobre la cobertura de gramíneas interactúa con variables ambientales. Por ejemplo, Berlow et al. (2003) estudiaron el efecto de la remoción de arbustos a través de un gradiente de humedad y encontraron que la cobertura de herbáceas fue generalmente mayor en los sitios intervenidos, con respecto al control, y que la misma se incrementó de forma lineal con la humedad del suelo. Esto indicaría que la respuesta de las gramíneas y herbáceas en el sitio de estudio de este trabajo requiere mayor tiempo, considerando las limitantes hídricas resultantes de precipitaciones anuales durante los años evaluados inferiores al valor promedio de la zona. Sin embargo, luego de 3 años de aplicados los cortes en fajas, la producción de materia seca del inter-coironal se triplicó respecto al testigo, aumento que se mantuvo en el tiempo. En forma similar, en un ambiente rolado en La Pampa, la productividad media del pastizal rolado fue $91 \%$ mayor que la del pastizal natural, como consecuencia del mejor aprovechamiento del agua edáfica por parte del estrato graminoso-herbáceo (Adema et al. 2003). Sin embargo, este tipo de manejo es incipiente en la región, no se ha aplicado a gran escala y actualmente el ganado continúa excluido del sitio de ensayo, por lo que no es posible corroborar la situación en las fajas bajo pastoreo. Asimismo, el efecto sobre la vegetación podría ser significativamente diferente de acuerdo al momento en el cual el ganado ingresa nuevamente a los arbustales manejados, ya sea inmediatamente o " $\mathrm{n}$ " años luego de realizados los cortes.

\section{Banco de semillas del suelo}

La emergencia de plántulas en condiciones óptimas de temperatura y humedad fue baja, considerando la cantidad de semillas viables reportadas para ambientes áridos y semiáridos (Bertiller and Aloia 1997; Bertiller 1998). Los valores obtenidos de la emergencia potencial reflejaron una gran heterogeneidad en el BSS, en concordancia con lo informado por otros autores (Thompson 1986; Aguiar and Sala 1997; Bertiller 1998). Si bien se encontraron algunas diferencias, no existió un efecto concreto de los tratamientos a lo largo del tiempo en la emergencia potencial. El BSS potencial de las fajas de $8 \mathrm{~m}$ fue mayor en la estación de primavera, resultados similares fueron hallados por Bertiller and Aloia (1997) para algunas semillas de especies dicotiledóneas perennes. Bonvissuto and Busso (2007) determinaron que la lluvia de semillas de Larrea divaricata se concentró entre diciembre y marzo, mientras que para Atriplex lampa se distribuyó a lo largo del año y en Jarava neaei se concentró en diciembre y enero. La distribución de la lluvia de semillas, afectó la emergencia potencial determinada por las mediciones realizadas en diciembre y 
marzo para el presente estudio. No obstante, la formación del BSS no es inmediata y depende de diversos factores, entre ellos los pulsos de producción de semillas, por lo cual el efecto de la aplicación de los tratamientos sobre el BSS podría notarse a mediano plazo. Además, la presencia de broza puede impedir el ingreso de semillas al BSS en los tratamientos de corte, principalmente de las semillas grandes que pueden quedar atrapadas. Si bien no se detectaron diferencias, el número de plántulas fue el doble (a partir de marzo de 2011) en las situaciones bajo arbustos respecto a entre arbustos. Resultados disímiles fueron también reportados en una estepa arbustiva de L. divaricata y A. lampa por Bonvissuto and Busso (2007), en donde la distribución de semillas de $A$. lampa fue homogénea en cuatro sitios ubicados dentro de los parches de vegetación, mientras que para las especies $L$. divaricata y $J$. neaei la distribución fue mayor entre parches de vegetación y en la periferia de los mismos. Sin embargo, es importante tener en cuenta que las semillas no necesariamente permanecen en esos lugares debido al movimiento lateral y otros tipos de dispersión secundaria (dirección y velocidad del viento) que ocurre en ambientes de escasa vegetación (Reichman 1984). Además, luego de la dispersión, las semillas podrían quedar retenidas en las pequeñas grietas existentes en la superficie del suelo, que hacen difícil la redistribución. Asimismo, es importante considerar la depredación de semillas por los ratones, insectos o aves. En los tratamientos de corte, la eliminación de la parte aérea puede reducir la cantidad de refugios (ratones) y sitios de percha (aves).

Al considerar el reclutamiento de plántulas in situ, de acuerdo con la teoría de la dinámica de parches (Sala and Aguiar 1995) cabría esperar que los arbustos en las zonas donde se aplican los cortes en fajas actúen como facilitadores, sumado al efecto protector de la broza en la superficie del suelo, que almacenaría humedad y podría actuar como una trampa de semillas. Si bien los arbustos compiten por los recursos con las gramíneas, la facilitación sería más importante que la competencia cuando los arbustos son pequeños. En el caso de los tratamientos de cortes en fajas, si bien los individuos son de pequeña altura luego del corte, las raíces son profundas por tratarse de individuos adultos, por lo que no competirían por el agua con las nuevas plántulas establecidas. La disminución de la amplitud térmica en los tratamientos de corte podría afectar de manera positiva el reclutamiento y establecimiento de plántulas que germinan en otoño.Sin embargo, no se encontraron diferencias entre tratamientos en el reclutamiento de plántulas, lo cual podría deberse a que la presencia de broza afectaría la germinación in situ al cambiar la calidad de la luz. Los valores hallados en el presente estudio (entre 1 y 55 plántulas $/ \mathrm{m}^{2}$ ) estuvieron dentro del rango reportado por Marone et al. (2000) en Mendoza, y son claramente inferiores a los que Bertiller and Coronato (1994) encontraron al evaluar sólo el banco de semillas germinable de la gramínea $F$. pallescens (i.e., densidades de hasta 600 plántulas $/ \mathrm{m}^{2}$ ). El aumento en el número total de plantas reclutadas en el último año de medición podría estar relacionado a la precipitación de marzo de 2012(28 mm), en comparación con los años previos en el mismo mes (3 y $4 \mathrm{~mm})$.

La emergencia potencial fue mayor que el reclutamiento de plantas in situ. Esta diferencia estaría relacionada, en parte, a la mortandad de semillas que logran germinar in situ, pero que no logran sobrevivir debido a las condiciones ambientales adversas, como la falta de humedad en el suelo, factor crítico en losecosistemasáridos (Reichenberger and Pyke 1990) al ser necesaria para la germinación de semillas, el establecimiento y la supervivencia de plántulas. Además, las semillas pueden ser consumidas por diversos organismos del suelo (insectos) y por las aves (Marone et al. 1998, 2000). En relación a la distribución espacial en el testigo, debido al efecto protector o de facilitación que ofrece la vegetación establecida (Sala and Aguiar 1995) se habría esperado una mayor densidad de plántulas en la situación bajo arbustos. No obstante, en el presente trabajo no se observaron diferencias en la cantidad de plántulas establecidas al comparar las situaciones bajo arbustos y entre arbustos en los dos años de estudio, obteniéndose diferencias significativas entre años. Para algunas especies como F. pallescens, Bertiller and Coronato (1994) encontraron que los micrositios determinados por el relieve fueron más importantes en el reclutamiento de plántulas, siendo mayor en micrositios ubicados a barlovento en laderas y a sotavento en zonas planas.

\section{CONCLUSIONES}

La capacidad de rebrote de los individuos de M. tridenspermitiría planificar la rotación delas fajas a lo largo del tiempo para la recuperación del arbustal. El efecto más importante de la incorporación de broza sobre la superficie del suelo se observó en la disminución de la 
amplitud térmica y en la humedad del suelo. Si bien la aplicación de los tratamientos de corte en fajas en el arbustal de $M$. tridens no afectó el banco de semillas (potencial) del suelo, ni el reclutamiento de plantas in situ, el aumento de producción forrajera del intercoironal y sus coberturas presentan resultados promisorios. En este sentido, el fin de aplicar técnicas de manejo de pastizales se encuentra directamente relacionado a la búsqueda de prácticas sustentables que permitan el mejoramiento de la producción animal. Los anchos de faja estudiados ( $4 \mathrm{~m}$ y $8 \mathrm{~m}$ ) inciden en la superficie con aporte de broza pero no son determinantes en la respuesta de la vegetación.

Agradecimientos. Al productor G. Sánchez de Ea Chali-Aike. A la Agencia de Extensión Río Gallegos del INTA, la UNPA y la Fundación Banco Santa Cruz por el financiamiento aportado.

\section{REFERENCIAS}

Adema, E. O., D. E. Buschiazzo, F. J. Babinec, T. Rucci, and V. F. Gómez Hermida. 2003. Balance de agua y productividad en un pastizal rolado en Chacharramendi, La Pampa. Publicación técnica No 50.Instituto Nacional de Tecnología Agropecuaria, Anguil, La Pampa, Argentina.

Aguiar, M. R., and O. E. Sala. 1997. Seed distribution constrains the dynamics of the Patagonian steppe. Ecology 78 (1):93-100.

Aguiar, M. R., and O. E. Sala. 1999. Patch structure, dynamics and implications for the functioning of arid ecosystems. Trends Ecol Evol 14(7):273-277.

Allegretti, L. I., C. B. Passera, and A. B. Robles. 1997. Short- and long- term effects of shrub management on vegetation in the Monte, Argentina. J Arid Environ 35:685-693.

Bahamonde, H. A., P. L. Peri, R. Álvarez, A. Barneix, A. Moretto, and G. Martínez Pastur. 2012. Litter decomposition and nutrients dynamics in Nothofagusantarctica forests under silvopastoral use in Southern Patagonia. Agroforest Syst 84(3):345-360.

Berlow, E. L., C. M. D'Antonio, and H. Swartz. 2003. Response of herbs to shrub removal across natural and experimental variation in soil moisture. Ecol Appl 13(5):1375-1387.

Bertiller, M. B. 1998. Spatial patterns of the germinable soil seed bank in northern Patagonia. Seed Sci Res 8(1):39-45.

Bertiller, M. B., and D. A. Aloia. 1997. Seed bank strategies in Patagonian semi-arid grasslands in relation to their management and conservation. Biodivers Conserv 6(4):639-650.

Bertiller, M. B., C. L. Sain, A. L. Carrera, and D. N. Vargas. 2005. Patterns of nitrogen and phosphorus conservation in dominant perennial grasses and shrubs across an aridity gradient in Patagonia, Argentina. J Arid Environ 62(2): 209-223.

Bertiller, M. B., and F. Coronato. 1994. Seed bank patterns of Festucapallescens in semiarid Patagonia (Argentina): a possible limit to bunch reestablishment. Biodivers Conserv 3:57-67.

Bonvissuto, G. L., and C. A. Busso. 2007. Seed rain in and between vegetation patches in arid Patagonia, Argentina. Phyton-Int J Exp Bot 76:47-59.

Covacevich, N., and H. Doberti. Efecto de la eliminación del matorral (Chiliotrichiumdiffusum) en el peso invernal de ovinos. Pp. 24 en: O. Strauch, N. Covacevich and A. Cárdenas (eds.). 2006. Manejo Sustentable de las praderas naturales de magallanes. Sochipa en Magallanes 30 años. Series Actas INIA N ${ }^{\circ}$ 35. Instituto de Investigaciones Agropecuarias, Punta Arenas, Región de Magallanes y de la Antártica Chilena, Chile.

Godagnone, R., andJ. C. Salazar Lea Plaza. 2004. Suelos de Santa Cruz. En: L. González and P. Rial (eds.). Guía Geográfica Interactiva Santa Cruz. Ediciones Instituto Nacional de Tecnología Agropecuaria, Santa Cruz, Argentina.

Golluscio, R., O. Sala, and W. Lauenroth. 1998. Differential use of large summer rainfall events by shrubs and grasses: a manipulative experiment in the Patagonian Steppe. Oecologia 115:17-25.

Gross, K. L. 1990. A comparison of methods for estimating seed numbers in the soil. J Ecol 78:1079-1093.

ISTA (International seed testing association). International rules for seed testing. 2009. The germination test. Bassersdorf, Switzerland.

Levy, E., and D. Madden. 1933. The point method of pasture analysis. New Zealand Journal of Agriculture 46:267269.

Marone, L., E. Bertilde, and M. E. Horno. 1998. Timing and spatial patterning of seed dispersal and redistribution in a South American warm desert. Plant Ecol 137:143-150.

Marone, L., M. E. Horno, and R. González del Solar. 2000. Post-Dispersal fate of seeds in the Monte Desert of Argentina: patterns of germination in successive wet and dry years. J Ecol 88:940-949.

Márquez, S., G. Fune, M. Cabildo, and E. Pucheta. 2002. Efectos del pastoreo sobre el banco de semillas germinable y la vegetación establecida en pastizales de montaña del centro de Argentina. Rev Chil Hist Nat 75(2):327-337.

Oliva, G., L. González, P. Rial, and E. Livraghi. 2001. El ambiente en la Patagonia Austral. Pp 19-82 en: P. Borrelli and G. Oliva (eds.). Ganadería sustentable en la Patagonia Austral. Ediciones Instituto Nacional de Tecnología Agropecuaria, Santa Cruz, Argentina.

Peri, P. L., E. Rivera, M. V. Sturzenbaum, D. Suárez, S. L. Billoni, J. Mora, B. Watson, and F. Milicevic. 2011. Water status of plants under strips management in shrub-grass steppe of Southern Patagonia. Actas IX International Rangeland Congress, Rosario, Santa Fe, Argentina. Pp. 651. 
Peri, P. L. 2011. Cortinas cortaviento en Patagonia sur: Revisión del conocimiento actual. $3^{\circ}$ Congreso Internacional de Salicáceas en Argentina. Neuquén, Neuquén, Argentina.

Reichenberger, G., and D. A. Pyke. 1990. Impact of early root competition on fitness components of four semiarid species. Oecologia 85(2):159-166.

Reichman, J. 1984. Spatial and temporal variation of seed distributions in Sonoran Desert soils. J Biogeogr 11:1-11.

Sala, O. E., and M. R. Aguiar. 1995. Origin, maintenance, and ecosystem effect of vegetation patches in arid lands. Fifth International Rangeland Congress, Salt Lake City, Utah, USA.

Sala, O. E., and W. K. Lauenroth. 1982. Small rainfall events: an ecological role in semiarid regions. Oecologia 53: 301-304.

Sharafatmandrad, M., A. Bahremand, M. Mesdaghi, and H. Barani. 2010. The role of rainfall and light interception by litter on maintenance of surface soil water content in an arid rangeland (Khabr National Park, southeast of Iran). Desert 15(1):53-60.

Simpson, R. L., M. A. Leck, and V. T. Parker. 1989. Seed Banks: general concepts and methodological issues. Pp. 3-8 en: M. A. Leck, V. T. Parker and R. L. Simpson (eds.). Ecology of Soil Seed Bank. Academic Press, California, USA.

Thompson, K. 1986. Small-scale heterogeneity in the seed bank of an acidic grassland. J Ecol 74:733-738.

Waring, R. H., and W. H. Schlesinger. 1985. Forest ecosystems: concepts and management. Academic Press, San Diego, California, USA.

Whitford, W. G., and E. L. Wade. 2002. Conceptual Framework and Paradigms. Pp. 1-19 en: W. G. Whitford (ed.). Ecology of Desert Systems. Academia Press, San Diego, California, USA. 